\title{
Human telomerase reverse transcriptase regulation by DNA methylation, transcription factor binding and alternative splicing (Review)
}

\author{
BRITTANY A. AVIN ${ }^{1,2}$, CHRISTOPHER B. UMBRICHT ${ }^{1,3,4}$ and MARTHA A. ZEIGER ${ }^{1,3}$ \\ Departments of ${ }^{1}$ Surgery, ${ }^{2}$ Graduate Program of Biochemistry Cellular and Molecular Biology, ${ }^{3}$ Oncology, \\ and ${ }^{4}$ Pathology, Johns Hopkins University School of Medicine, Baltimore, MD 21205, USA
}

Received September 10, 2016; Accepted October 17, 2016

DOI: $10.3892 /$ ijo.2016.3743

\begin{abstract}
The catalytic subunit of telomerase, human telomerase reverse transcriptase (hTERT), plays an essential role in telomere maintenance to oppose cellular senescence and, is highly regulated in normal and cancerous cells. Regulation of hTERT occurs through multiple avenues, including a unique pattern of $\mathrm{CpG}$ promoter methylation and alternative splicing. Promoter methylation affects the binding of transcription factors, resulting in changes in expression of the gene. In addition to expression level changes, changes in promoter binding can affect alternative splicing in a cotranscriptional manner. The alternative splicing of hTERT results in either the full length transcript which can form the active telomerase complex with hTR, or numerous inactive isoforms. Both regulation strategies are exploited in cancer to activate telomerase, however, the exact mechanism is unknown. Therefore, unraveling the link between promoter methylation status and alternative splicing for hTERT could expose yet another level of hTERT regulation. In an attempt to provide insight into the cellular control of active telomerase in cancer, this review will discuss our current perspective on $\mathrm{CpG}$ methylation of the hTERT promoter region, summarize the different forms of alternatively spliced variants, and examine examples of transcription factor binding that affects splicing.
\end{abstract}

\section{Contents}

1. Telomeres and telomerase

2. Telomerase regulation

3. Gene regulation by methylation

4. hTERT promoter methylation in cancer

Correspondence to: Dr Martha A. Zeiger, Department of Surgery, Johns Hopkins Hospital, 600 N. Wolfe Street, Blalock 606, Baltimore, MD 21287, USA

E-mail: mzeiger@jhmi.edu

Key words: TERT, alternative splicing, promoter, DNA methylation, transcription, cancer
5. Transcription factor regulation by methylation of hTERT promoter

6. Role of transcription factor binding on splicing

7. Telomerase regulation by alternative splicing in hTERT

8. Concluding remarks

\section{Telomeres and telomerase}

Each round of DNA replication results in the shortening of DNA strands due to the inability of the replication complex to completely replicate the lagging strand. This process in turn eventually results in genomic instability and loss of genetic information after multiple rounds of replication. To solve this end-replication problem, chromosome ends are capped by telomeres, which consist of six nucleotide repeats and specialized binding proteins that buffer replication losses (1). The repeats can be regenerated by the telomerase enzyme complex, which consists of a non-coding RNA, hTR, serving as the hexamer repeat template, and the catalytic subunit, reverse transcriptase (hTERT). By counteracting the telomere shortening incurred by the DNA replication process, the telomerase complex lengthens telomeres, thereby prolonging cell survival and allowing continued proliferation (2).

\section{Telomerase regulation}

Telomerase activity is low to absent in somatic cells, though highly expressed in embryonic and stem cells. Telomerase is also upregulated in cancer, as over $90 \%$ of human malignancies show telomerase expression, considered an early event in cancer progression $(3,4)$. In cancer, the main mechanism of telomerase activation is through regulation of hTERT transcription, through genetic changes such as mutations altering transcription factor binding, by epigenetic changes such as histone modification and chromatin remodeling or promoter methylation, and by alternative splicing of the transcript (2,5-7). As explored below, a connection between the promoter methylation and the alternative splicing is emerging in the field as a means by which cancer cells turn on hTERT expression, resulting both in active telomerase and telomere elongation. 


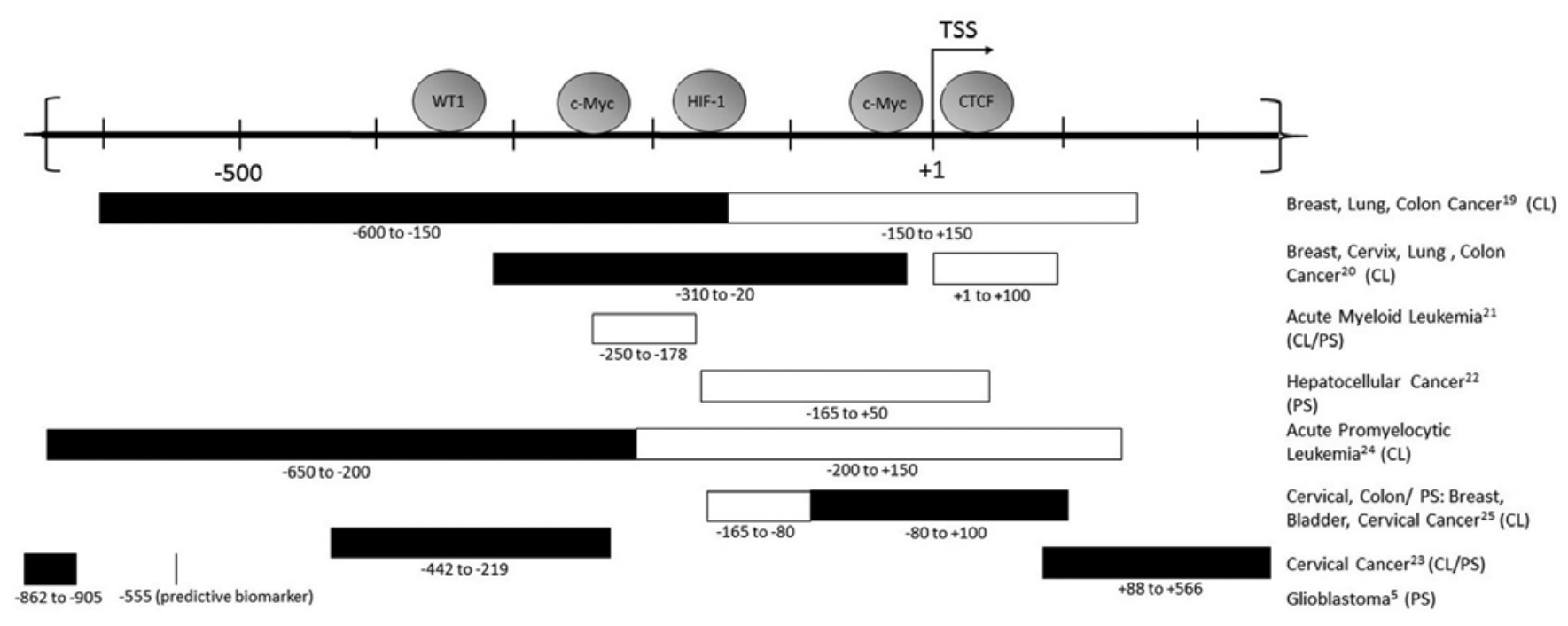

Figure 1. hTERT promoter exhibits a distinct methylation pattern in cancer cells positive for telomerase activity. hTERT promoter is -1876 to +335 relative to the transcription start site (TSS), with section -600 to +200 depicted above. Transcription factors (WT1, c-Myc, HIF-1, CTCF) and their relative binding sites at the promoter are shown. The methylation status of the hTERT promoter in several cell lines (CL) and/or patient blood or tumor samples (PS) are shown, with black boxes indicating methylated $\mathrm{CpG}$ sites, and white boxes indicating unmethylated $\mathrm{CpG}$ sites. In glioblastoma the CpG site at -555 is methylated.

\section{Gene regulation by methylation}

In higher eukaryotes, DNA methylation at $\mathrm{CpG}$ sites in and around gene promoter regions controls and regulates gene expression. CpG methylation is directed by DNA methyltransferases (DNMTs) which methylate the fifth carbon of the pyrimidine ring of cytosine (8). The principal methyltransferase, DNMT1, adds methyl groups during DNA replication and de novo methylates DNA in cancer (9). Many CpG sites are clustered into $\mathrm{CpG}$ islands that are typically 1,000 base pairs long and have high GC content. Approximately $80 \%$ of $\mathrm{CpG}$ sites are methylated in mammals, largely in intergenic regions known as heterochromatin, while most sites in promoters and first exons remain unmethylated $(10,11)$. The promoter and transcription start site (TSS) tends to be unmethylated in actively transcribed genes, since methylated DNA is associated with gene silencing through both interference of transcription factor binding and, by affecting chromatin architecture (12). Importantly, aberrant DNA methylation is a hallmark of cancer cells, and tends to occur early in cancer development (13). In cancer, characteristic changes in methylation patterns involve both genome-wide CpG hypomethylation, which occurs predominantly in intergenic regions, and hypermethylation of $\mathrm{CpG}$ islands at promoters. Promoter hypermethylation may result in silencing of tumor suppressors, and promoter hypomethylation can result in activation of proto-oncogenes (14). Intergenic hypomethylation may also lead to expression of dormant non-coding RNA species and otherwise suppressed genetic elements transcribed from normally silent regions of the genome (15-17).

\section{4. hTERT promoter methylation in cancer}

The hTERT promoter is located in a $4 \mathrm{~kb} \mathrm{CpG}$ island -1800 to +2200 (relative to TSS), and has a GC content of $70 \%$ (18). The precise pattern of promoter methylation that results in activation of hTERT in cancer is still under investigation. However, a methylation pattern does emerge from many studies of the promoter, including extensive bisulfite sequencing of telomerase positive cancer cell lines. The promoter region of the actively transcribed hTERT is demethylated at the TSS [-200 to +100 ], while the promoter region further upstream of the TSS [-650 to -200] is hypermethylated (19). Studies examining specific sections of the hTERT promoter corroborate this in a myriad of cancer cell lines as well as hematological malignancies and solid tumor types, as depicted in Fig. 1 (5,19-26). Its methylation status is also considered a biomarker; in pediatric brain tumors, one methylated $\mathrm{CpG}$ site in the promoter (cg11625005) is used as a reliable marker for tumor progression and prognosis (5).

\section{Transcription factor regulation by methylation of hTERT promoter}

It is known that the hTERT methylation pattern plays a major role in transcription factor binding, which in turn alters the expression of hTERT. This is demonstrated by experimental evidence pointing to a 'minimal promoter' corresponding to transcription factor binding sites, e.g. c-Myc for hTERT at -258 to -78 , that must be unmethylated in order for hTERT expression to occur (27). Methylation also plays a significant role further upstream in the hTERT promoter where many repressor binding sites are located. These sites are hypermethylated in cancer to prevent binding of repressors such as the Wilms' tumor protein (WT1) and the transcriptional repressor CCCTC-binding factor (CTCF) that binds to $\mathrm{CpG}$ rich regions at the TSS $(21,25,27-29)$. Perturbing the methylation status of the promoter with 5aza-2'-deoxycytidine (DAC), which globally reduces DNA methylation by DNMT inhibition, results in reduced levels of hTERT transcription, which may be due to demethylation of the repressor binding sites (30).

$c-M y c$. Hypomethylation at the minimal promoter of hTERT allows c-Myc, a key positive regulator of hTERT expression, 
to bind 242 bases upstream of the TSS at the E-box. Studies have shown that c-Myc binding is methylation-sensitive and that binding is greatly reduced or absent when the site is methylated (31). This control of hTERT expression by E-box methylation is also seen in human embryonic teratocarcinoma cells. The undifferentiated embryonic cells have higher expression of hTERT and telomerase activity with hypomethylation of the minimal promoter. Conversely, during the process of differentiation of these cells there is a significant increase in methylation at the E-box, resulting in inactivation of hTERT expression. This mode of repression of hTERT can be reversed by treatment of late differentiating cells with DAC, highlighting the importance of c-Myc binding and regulation by methylation (27).

WT1. In contrast to the minimal promoter being hypomethylated to allow c-Myc binding, the promoter region further upstream, corresponding to the binding sites of multiple repressors, is hypermethylated in cancer. For example, the repressor WT1, with its binding site from -358 to -349 , is known to suppress hTERT transcription (27). This is supported by a study in clear cell renal cell carcinoma, in which WT1 is overexpressed, and direct binding of WT1 to the promoter results in repression of hTERT (32). WT1 binding is also known to be methylation sensitive, with binding interference assays showing reduced binding when one or more methylated bases are present in the binding sequence (33). The WT1 binding site of hTERT exhibits increased $\mathrm{CpG}$ methylation in cancer, resulting in blocking the repressive effects of the factor for hTERT expression $(20,34)$.

CTCF. Similar to WT1, as a repressor of hTERT, CTCF binds adjacent to the transcription start site, near the beginning of exon one $(+4$ to +39$)$, and near the beginning of exon two $(+422$ to +440$)$. Studies have shown that CTCF binding at the first site represses hTERT transcription and that this binding is blocked in cancer (35). Blocking is established by methylation as CTCF's binding affinity is inversely correlated with the degree of methylation; CTCF is unable to bind fully methylated DNA. Cancer cells have aberrant methylation in the first CTCF binding site, typified by HPV-transformed cervical cancer cells showing increased methylation and activated hTERT expression (23). Conversely, in colon cancer cell lines, downregulating DNTM1, and the resultant demethylation of the CTCF binding site, causes increased CTCF binding and repression of hTERT $(36,37)$. Furthermore, in breast cancer cells, hTERT transcript levels increase with a concomitant decrease in cellular apoptosis when CTCF is downregulated by siRNA (38).

\section{Role of transcription factor binding on splicing}

While it is known that transcription factors binding to the promoter regulate gene expression, it is becoming evident that such binding also affects splicing. Proteins with a cis regulatory role in both transcription and splicing have been demonstrated in multiple genes. The regulation is believed to act through the kinetic coupling model, where the rate of transcription elongation affects alternative splicing $(39,40)$. Experiments assessing alternative splicing in a promoter swapping system have established promoter specificity in controlling alternative splicing (41). This splicing role is consistent with splicing occurring co-transcriptionally as splicing factor assembly arises during transcription. As detailed below, manipulation of splicing through transcription factors can be accomplished either by the factor directly influencing the spliceosome or, by recruitment of additional factors $(42,43)$.

Direct splicing role of WT1 with $U 2 A F$ and RBM4. WT1, a negative regulator of hTERT, promotes splicing of multiple transcripts by both interacting directly with several splicing factors and through incorporation into spliceosomes. In the nucleus, the regulator co-localizes with the splicing machinery, specifically with U2AF and RBM4. The U2AF heterodimer is essential for binding upstream of the splice site and helps the U2 snRNA anneal at the branch point. Interaction with WT1 affects U2AF binding and therefore splice site selection (44). Also in splice site selection, RBM4 influences alternative splicing during selection of the 5' exon splice site, and interacts with WT1 in nuclear speckles, thought to be compartments for spliceosome assembly (45). Recruitment of these splicing factors by WT1 highlights the link between the transcription factor binding and spliceosome assembly.

Indirect splicing role of WT1 with SRPK1. While WT1 interacts with the splicing machinery as discussed above, it also plays an indirect role in splicing by transcriptionally repressing SRPK1, a splicing factor kinase. As shown in acute myeloid leukemia, where it is often overexpressed, WT1 causes alternative exon usage events commonly seen in the disease (46). Conversely, when WT1 is knocked down in hematopoietic progenitor cells, vascular endothelial growth factor (VEGF) exhibits an atypical splicing pattern (47). In studying the relationship between WT1 and VEGF, an important growth factor in cancer, loss of WT1 results in increased abundance of isoform VEGF-a120, while transfection of WT1 results in exon inclusion and loss of the 120 isoform. This demonstrates that WT1 is essential for controlling the splicing of VEGF-a (48). Studies of the VEGF-b isoform have also shown alteration of splicing patterns with WT1 manipulation. Transfection of various cell lines with WT1 results in an increase of VEGF165b. Furthermore, inclusion of the WT1 KTS sequence, which plays a role in RNA binding, shows no effect on splicing, while a single nucleotide change that alters DNA binding of WT1 changes the splicing of VEGF. Thus, splicing correlates with the ability of the transcription factor WT1 to stably bind DNA, rather than WT1 acting post-transcriptionally (49).

Splicing role of HIF-1 and hypoxia on hTERT. Hypoxia is known to enhance hTERT expression through activation of the hTERT promoter in stem cells as well as in cancer $(50,51)$. In hypoxic tumor conditions, HIF-1 is overexpressed and activates the hTERT promoter by binding at two sites between -165 and +51 . If the HIF-1 binding sites are mutated, hTERT promoter activity decreases even in normoxic conditions and under hypoxic conditions, the HIF-1 induced overexpression of hTERT is lost. It has been shown that in hypoxia, cell survival is increased through maintenance of an undifferentiated state, and this correlates with increased hTERT expression and telomerase activity compared to normoxic conditions $(51,52)$. 

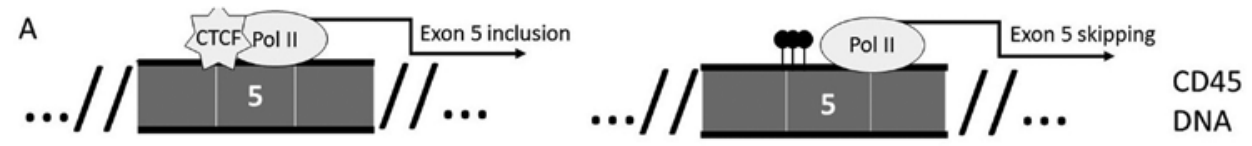

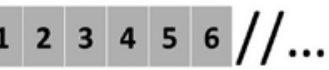

B
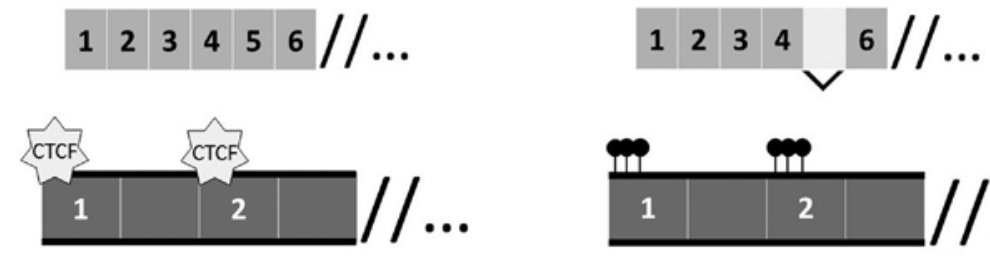

mRNA
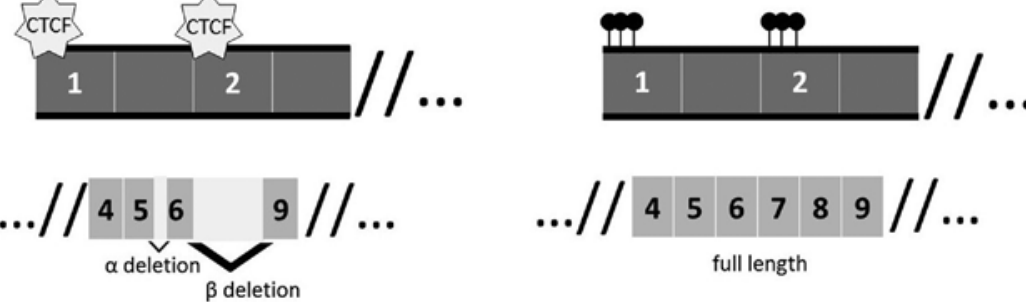

* hTERT
DNA

${ }^{*}$ mRNA

Figure 2. Intragenic binding of CTCF affects transcript splicing. (A) The well-established mechanism of exon 5 inclusion in CD45. Binding of exon 5 by CTCF to its unmethylated binding site causes pausing of polymerase II and inclusion of exon 5 in the final mRNA. When the site is methylated, CTCF cannot bind and the final transcript skips exon 5. (B) Hypothetical (*) mechanism of long range CTCF interactions influencing hTERT splicing. Common exon deletions in hTERT splice variants with the CTCF binding sites at exon 1 and exon 2. CTCF binding at exon 1 is known to affect hTERT expression, while its potential role in splicing is unknown.

Furthermore, the splicing pattern of hTERT is quite variable at differing levels of oxygenation. Overall, the hTERT splicing pattern is altered in hypoxia, with an increased expression of the full length active form, while the $\beta$ deletion and $\alpha / \beta$ double deletion splice variants remain unchanged. Additionally, blocking $\alpha$ and $\beta$ splicing results in spontaneous human embryonic stem cell differentiation (50). In hypoxia, HIF-1 increases the association of RNA Pol II with transcription initiation factors, resulting in more effective and efficient transcription. It has been proposed that modulation of hTERT transcription by HIF-1 in hypoxia controls splicing by affecting Pol II rates of transcription and enhancing expression of the active full length transcript (53).

Direct splicing role of CTCF intragenic binding with Pol II pausing. The direct role of CTCF modulating alternative splicing in a co-transcriptional manner has recently emerged. Shukla and colleagues (56) showed that CTCF binding at actively transcribed DNA causes RNA polymerase II pausing, resulting in recognition of weak splice site signals and therefore inclusion of 'weak' exons in CD45. They also demonstrated that the pausing was methylation dependent; when CTCF cannot bind the methylated site, weak exons are not included in the final spliced transcript (Fig. 2A) $(55,56)$. Multiple additional genes have been shown to have promoterproximal CTCF binding (100 bases downstream of the TSS) that results in longer Pol II pausing compared to genes without promoter-proximal binding sites. This effect is dependent on the binding site position relative to the TSS, with less pausing associated with sites at greater distance from the TSS (57). RNA polymerase II pausing due to CTCF results in decreased processivity and contributes to promoter proximal pausing, thereby directly modulating the dynamics of transcription and splicing $(57,58)$.

Indirect splicing role of CTCF by facilitation of long range interactions. CTCF is well known for its architectural role in establishing boundaries based on topologically associating domains (TADs) that link distant enhancers with promoters and other regulatory sequences. This chromatin looping by CTCF allows for architectural reorganization resulting in co-location of factors to influence transcription. Indeed, approximately $15 \%$ of CTCF binding sites are located in promoters, while over $40 \%$ of CTCF binding sites are located in the 5'UTR, introns, and other intragenic regions. For example, the murine Myb locus has a CTCF binding site in the first intron, which loops to the promoter during differentiation, and a second CTCF site in a regulatory element further upstream. This CTCF interaction allows for juxtaposition of necessary transcription factors to regulate Pol II and modulate expression of Myb. After differentiation, the expression of the gene is halted due to CTCF architecture reorganization. This highlights the ability of CTCF to regulate initiation of transcription as well as elongation and Pol II pausing during transcription by looping together promoter, upstream enhancer elements and intronic sequences. Influencing the elongation of Pol II, as well as the juxtaposition of regulatory factors, are known to be important for splicing, as discussed above regarding the elongation rate of Pol II and CD45 splicing $(59,60)$.

\section{Telomerase regulation by alternative splicing in hTERT}

While in many genes transcription factor binding has been shown to play a role in splicing, the mechanism of hTERT splicing is not as clearly defined. hTERT splicing plays a crucial role in dictating the activity of telomerase, since only the full-length transcript is catalytically active in the telomerase ribonucleoprotein complex. Furthermore, many cancers show changes in hTERT splicing patterns.

hTERT splicing switch in cancer. Modulation of alternative splicing is detected in multiple types of cancers, where cancer cells utilize an alternative splicing switch that results in discernible isoform signatures (61). This switching is nonrandom and also seen in tissue development, including the hTERT splice switch, well characterized in kidney develop- 
ment and various cancer cell types (62-74). This mode of hTERT regulation has been proposed to be necessary due to the difficulty to completely cease transcription, since even very small amounts of transcript may have significant cellular effects, given the limited number of cellular targets, i.e., 92 telomeres in the normal human cell (2). Cancer cells with active telomerase have an average of 20 hTERT transcripts per cell resulting in 100-500 active telomerase complexes (75). Alternative splicing yielding inactive and/or inhibitory forms of hTERT allows for downregulation of telomerase activity without complete repression of transcription.

Mechanism of hTERT alternative splicing. Studies on the mechanisms of alternative splicing of hTERT so far have revealed regulation primarily by long range interactions, not at nearby splice sites as seen in other genes. One proposed mechanism is via variable number tandem repeats (VNTR), that are located in intronic regions over $1 \mathrm{~kb}$ from the splice site, regulating splicing of the $\beta$ deletion form. The mechanism of VNTR control over splicing is not understood, but could involve recruitment of RNA binding factors to the repeats. These RNA binding factors would then interact and form a specific landscape with other proteins conducive to spliceosome recruitment $(2,75)$. The mechanism for the dependence on VNTR for splicing of the $\beta$ deletion needs further examination, as do additional splicing mechanisms for the other isoforms.

Over twenty different isoforms of hTERT have so far been reported, with the most common being various deletions in the reverse transcriptase domain, such as the $\alpha$ deletion, $\beta$ deletion and $\alpha / \beta$ deletion shown in Fig. 2B (76). Other important isoforms have been identified, such as intron 2 and 14 retention in lung and colon cancer as well as exclusion of exon 2 in normal cells (77,78). All known isoforms result in an inactive telomerase complex. For example, the $\alpha$ deletion is a dominant negative variant while the $\beta$ deletion results in a truncated protein targeted for nonsense-mediated decay (75). While normal cells express mainly inactive hTERT isoforms, a splicing switch occurs in cancer cells, resulting in production of the full length active transcript (79). The cause of the splicing switch is unknown, but is likely due to changes of binding factors, possibly similar to changes to long range interactions through VNTR in the case of the $\beta$ deletion or, as stated above, from the ability of certain transcription factors to bind and affect transcription elongation rates.

\section{Concluding remarks}

Human telomerase reverse transcriptase expression has many facets of regulation, including promoter methylation and alternative splicing, as outlined above. These two methods of regulation can become intertwined by the co-transcriptional nature of mammalian pre-mRNA splicing. This allows transcription factors bound to the gene, including the promoter, to influence alternative splicing of the transcript. Understanding the regulation of hTERT is crucial to understanding telomerase activity in normal cells as well as in cancer. Elucidating the mechanism of cellular control of hTERT transcription will further our knowledge of the intricate instructions directing activation of this essential genome maintenance machinery.
Study of hTERT transcription is a unique opportunity to understand the control of a low abundance transcript with a prominent role in cancer. Insight into how cancer commandeers the regulatory control, be it through methylation or through the splicing machinery, to produce full length hTERT and resulting in active telomerase would be highly beneficial clinically. Currently, therapeutic telomerase inhibitors are not well tolerated by patients. The therapy must be continually administered for multiple replication cycles to have an effect, resulting in the need for extended periods of treatment, which can be challenging due to significant drug toxicity. Promising anticancer therapeutics such as imetelstat, an antisense oligonucleotide binding to hTR, target the active telomerase complex, or consist of small molecule inhibitors inhibiting active TERT binding to the RNA (80). Alternatively, therapies inhibiting TERT from being activated before the telomerase complex forms, by altered expression or splicing, could provide a new mechanistic avenue for effective anticancer therapeutics.

\section{References}

1. Lü MH, Liao ZL, Zhao XY, Fan YH, Lin XL, Fang DC, Guo H and Yang SM: hTERT-based therapy: A universal anticancer approach (Review). Oncol Rep 28: 1945-1952, 2012.

2. Wong MS, Chen L, Foster C, Kainthla R, Shay JW and Wright WE: Regulation of telomerase alternative splicing: A target for chemotherapy. Cell Rep 3: 1028-1035, 2013.

3. Koziel JE, Fox MJ, Steding CE, Sprouse AA and Herbert BS: Medical genetics and epigenetics of telomerase. J Cell Mol Med 15: 457-467, 2011.

4. Umbricht CB, Sherman ME, Dome J, Carey LA, Marks J, Kim N and Sukumar S: Telomerase activity in ductal carcinoma in situ and invasive breast cancer. Oncogene 18: 3407-3414, 1999.

5. Castelo-Branco P, Choufani S, Mack S, Gallagher D, Zhang C, Lipman T, Zhukova N, Walker EJ, Martin D, Merino D, et al: Methylation of the TERT promoter and risk stratification of childhood brain tumours: An integrative genomic and molecular study. Lancet Oncol 14: 534-542, 2013.

6. Liu X, Bishop J, Shan Y, Pai S, Liu D, Murugan AK, Sun H, El-Naggar AK and Xing M: Highly prevalent TERT promoter mutations in aggressive thyroid cancers. Endocr Relat Cancer 20: 603-610, 2013.

7. Kyo S, Takakura M, Fujiwara T and Inoue M: Understanding and exploiting hTERT promoter regulation for diagnosis and treatment of human cancers. Cancer Sci 99: 1528-1538, 2008.

8. Adams RL: Eukaryotic DNA methyltransferases - structure and function. BioEssays 17: 139-145, 1995.

9. Jair KW, Bachman KE, Suzuki H, Ting AH, Rhee I, Yen RW, Baylin SB and Schuebel KE: De novo CpG island methylation in human cancer cells. Cancer Res 66: 682-692, 2006.

10. Wan J, Oliver VF, Wang G, Zhu H, Zack DJ, Merbs SL and Qian J: Characterization of tissue-specific differential DNA methylation suggests distinct modes of positive and negative gene expression regulation. BMC Genomics 16: 49, 2015.

11. Deaton $\mathrm{AM}$ and Bird A: $\mathrm{CpG}$ islands and the regulation of transcription. Genes Dev 25: 1010-1022, 2011.

12. Baylin SB and Jones PA: A decade of exploring the cancer epigenome - biological and translational implications. Nat Rev Cancer 11: 726-734, 2011.

13. Stirzaker C, Millar DS, Paul CL, Warnecke PM, Harrison J, Vincent PC, Frommer M and Clark SJ: Extensive DNA methylation spanning the $\mathrm{Rb}$ promoter in retinoblastoma tumors. Cancer Res 57: 2229-2237, 1997.

14. Smith IM, Glazer CA, Mithani SK, Ochs MF, Sun W, Bhan S, Vostrov A, Abdullaev Z, Lobanenkov V, Gray A, et al: Coordinated activation of candidate proto-oncogenes and cancer testes antigens via promoter demethylation in head and neck cancer and lung cancer. PLoS One 4: e4961, 2009.

15. Jones PA and Baylin SB: The fundamental role of epigenetic events in cancer. Nat Rev Genet 3: 415-428, 2002. 
16. Tahira AC, Kubrusly MS, Faria MF, Dazzani B, Fonseca RS, Maracaja-Coutinho V, Verjovski-Almeida S, Machado MC and Reis EM: Long noncoding intronic RNAs are differentially expressed in primary and metastatic pancreatic cancer. Mol Cancer 10: 141, 2011.

17. Hangauer MJ, Vaughn IW and McManus MT: Pervasive transcription of the human genome produces thousands of previously unidentified long intergenic noncoding RNAs. PLoS Genet 9: e1003569, 2013.

18. Choi JH, Park SH, Park J, Park BG, Cha SJ, Kong KH, Lee KH and Park AJ: Site-specific methylation of $\mathrm{CpG}$ nucleotides in the hTERT promoter region can control the expression of hTERT during malignant progression of colorectal carcinoma. Biochem Biophys Res Commun 361: 615-620, 2007.

19. Zinn RL, Pruitt K, Eguchi S, Baylin SB and Herman JG hTERT is expressed in cancer cell lines despite promoter DNA methylation by preservation of unmethylated DNA and active chromatin around the transcription start site. Cancer Res 67: 194-201, 2007.

20. Guilleret I and Benhattar J: Unusual distribution of DNA methylation within the hTERT CpG island in tissues and cell lines. Biochem Biophys Res Commun 325: 1037-1043, 2004.

21. Pettigrew KA, Armstrong RN, Colyer HA, Zhang SD, Rea IM, Jones RE, Baird DM and Mills KI: Differential TERT promoter methylation and response to 5-aza-2'-deoxycytidine in acute myeloid leukemia cell lines: TERT expression, telomerase activity, telomere length, and cell death. Genes Chromosomes Cancer 51: 768-780, 2012.

22. Iliopoulos D, Satra M, Drakaki A, Poultsides GA and Tsezou A: Epigenetic regulation of hTERT promoter in hepatocellular carcinomas. Int J Oncol 34: 391-399, 2009.

23. de Wilde J, Kooter JM, Overmeer RM, Claassen-Kramer D, Meijer CJ, Snijders PJ and Steenbergen RD: hTERT promoter activity and $\mathrm{CpG}$ methylation in HPV-induced carcinogenesis. BMC Cancer 10: 271, 2010.

24. Azouz A, Wu YL, Hillion J, Tarkanyi I, Karniguian A, Aradi J, Lanotte M, Chen GQ, Chehna M and Ségal-Bendirdjian E: Epigenetic plasticity of hTERT gene promoter determines retinoid capacity to repress telomerase in maturation-resistant acute promyelocytic leukemia cells. Leukemia 24: 613-622, 2010.

25. Renaud S, Loukinov D, Abdullaev Z, Guilleret I, Bosman FT, Lobanenkov V and Benhattar J: Dual role of DNA methylation inside and outside of CTCF-binding regions in the transcriptional regulation of the telomerase hTERT gene. Nucleic Acids Res 35: 1245-1256, 2007.

26. Kumari A, Srinivasan R, Vasishta RK and Wig JD: Positive regulation of human telomerase reverse transcriptase gene expression and telomerase activity by DNA methylation in pancreatic cancer. Ann Surg Oncol 16: 1051-1059, 2009.

27. Lopatina NG, Poole JC, Saldanha SN, Hansen NJ, Key JS, Pita MA, Andrews LG and Tollefsbol TO: Control mechanisms in the regulation of telomerase reverse transcriptase expression in differentiating human teratocarcinoma cells. Biochem Biophys Res Commun 306: 650-659, 2003.

28. Zhu J, Zhao Y and Wang S: Chromatin and epigenetic regulation of the telomerase reverse transcriptase gene. Protein Cell 1: 22-32, 2010.

29. Guilleret I and Benhattar J: Demethylation of the human telomerase catalytic subunit (hTERT) gene promoter reduced hTERT expression and telomerase activity and shortened telomeres. Exp Cell Res 289: 326-334, 2003.

30. Tsujioka T, Yokoi A, Itano Y, Takahashi K, Ouchida M, Okamoto S, Kondo T, Suemori S, Tohyama Y and Tohyama K: Five-aza-2'-deoxycytidine-induced hypomethylation of cholesterol 25-hydroxylase gene is responsible for cell death of myelodysplasia/leukemia cells. Sci Rep 5: 16709, 2015.

31. Prendergast G and Ziff E: Methylation-sensitive sequencespecific DNA binding by the c-Myc basic region. Science 251: 186-189, 1991.

32. Sitaram RT, Degerman S, Ljungberg B, Andersson E, Oji Y, Sugiyama H, Roos G and Li A: Wilms' tumour 1 can suppress hTERT gene expression and telomerase activity in clear cell renal cell carcinoma via multiple pathways. Br J Cancer 103: 1255-1262, 2010.

33. Drummond IA, Rupprecht HD, Rohwer-Nutter P, LopezGuisa JM, Madden SL, Rauscher FJ III and Sukhatme VP: DNA recognition by splicing variants of the Wilms' tumor suppressor, WT1. Mol Cell Biol 14: 3800-3809, 1994.
34. Shin KH, Kang MK, Dicterow E and Park NH: Hypermethylation of the hTERT promoter inhibits the expression of telomerase activity in normal oral fibroblasts and senescent normal oral keratinocytes. Br J Cancer 89: 1473-1478, 2003.

35. Renaud S, Loukinov D, Bosman FT, Lobanenkov V and Benhattar J: CTCF binds the proximal exonic region of hTERT and inhibits its transcription. Nucleic Acids Res 33: 6850-6860, 2005.

36. Feldmann A, Ivanek R, Murr R, Gaidatzis D, Burger L and Schübeler D: Transcription factor occupancy can mediate active turnover of DNA methylation at regulatory regions. PLoS Genet 9: e1003994, 2013.

37. Choi JH, Min NY, Park J, Kim JH, Park SH, Ko YJ, Kang Y, Moon YJ, Rhee S, Ham SW, et al: TSA-induced DNMT1 downregulation represses hTERT expression via recruiting CTCF into demethylated core promoter region of hTERT in HCT116. Biochem Biophys Res Commun 391: 449-454, 2010.

38. Meeran SM, Patel SN and Tollefsbol TO: Sulforaphane causes epigenetic repression of hTERT expression in human breast cancer cell lines. PLoS One 5: e11457, 2010.

39. Kornblihtt AR: Promoter usage and alternative splicing. Curr Opin Cell Biol 17: 262-268, 2005.

40. Nieto Moreno N, Giono LE, Cambindo Botto AE, Muñoz MJ and Kornblihtt AR: Chromatin, DNA structure and alternative splicing. FEBS Lett 589: 3370-3378, 2015.

41. Cramer P, Cáceres JF, Cazalla D, Kadener S, Muro AF, Baralle FE and Kornblihtt AR: Coupling of transcription with alternative splicing: RNA pol II promoters modulate SF2/ASF and 9G8 effects on an exonic splicing enhancer. Mol Cell 4: 251-258, 1999.

42. Schor IE, Gómez Acuña LI and Kornblihtt AR: Coupling between transcription and alternative splicing. Cancer Treat Res 158: 1-24, 2013.

43. Naftelberg S, Schor IE, Ast G and Kornblihtt AR: Regulation of alternative splicing through coupling with transcription and chromatin structure. Annu Rev Biochem 84: 165-198, 2015.

44. Davies RC, Calvio C, Bratt E, Larsson SH, Lamond AI and Hastie ND: WT1 interacts with the splicing factor U2AF65 in an isoform-dependent manner and can be incorporated into spliceosomes. Genes Dev 12: 3217-3225, 1998.

45. Markus MA, Heinrich B, Raitskin O, Adams DJ, Mangs H, Goy C, Ladomery M, Sperling R, Stamm S and Morris BJ: WT1 interacts with the splicing protein RBM4 and regulates its ability to modulate alternative splicing in vivo. Exp Cell Res 312: 3379-3388, 2006.

46. Mohamed AM, Balsat M, Thenoz M, Koering C, Payen-Gay L, Cheok M, Mortada H, Auboeuf D, Pinatel C, El-Hamri M, et al: Oncogene- and drug resistance-associated alternative exon usage in acute myeloid leukemia (AML). Oncotarget 7: 2889-2909, 2016.

47. Katuri V, Gerber S, Qiu X, McCarty G, Goldstein SD, Hammers H, Montgomery E, Chen AR and Loeb DM: WT1 regulates angiogenesis in Ewing Sarcoma. Oncotarget 5: 2436-2449, 2014.

48. Cunningham TJ, Palumbo I, Grosso M, Slater N and Miles CG: WT1 regulates murine hematopoiesis via maintenance of VEGF isoform ratio. Blood 122: 188-192, 2013.

49. Amin EM, Oltean S, Hua J, Gammons MV, Hamdollah-Zadeh M, Welsh GI, Cheung MK, Ni L, Kase S, Rennel ES, et al: WT1 mutants reveal SRPK1 to be a downstream angiogenesis target by altering VEGF splicing. Cancer Cell 20: 768-780, 2011.

50. Radan L, Hughes CS, Teichroeb JH, Vieira Zamora FM, Jewer M, Postovit LM and Betts DH: Microenvironmental regulation of telomerase isoforms in human embryonic stem cells. Stem Cells Dev 23: 2046-2066, 2014

51. Yatabe N, Kyo S, Maida Y, Nishi H, Nakamura M, Kanaya T, Tanaka M, Isaka K, Ogawa S and Inoue M: HIF-1-mediated activation of telomerase in cervical cancer cells. Oncogene 23: 3708-3715, 2004.

52. Nishi H, Nakada T, Kyo S, Inoue M, Shay JW and Isaka K: Hypoxia-inducible factor 1 mediates upregulation of telomerase (hTERT). Mol Cell Biol 24: 6076-6083, 2004.

53. Anderson CJ, Hoare SF, Ashcroft M, Bilsland AE and Keith WN: Hypoxic regulation of telomerase gene expression by transcriptional and post-transcriptional mechanisms. Oncogene 25: 61-69, 2006.

54. Kechris K, Yang YH and Yeh RF: Prediction of alternatively skipped exons and splicing enhancers from exon junction arrays. BMC Genomics 9: 551, 2008.

55. Kornblihtt AR: CTCF: From insulators to alternative splicing regulation. Cell Res 22: 450-452, 2012. 
56. Shukla S, Kavak E, Gregory M, Imashimizu M, Shutinoski B, Kashlev M, Oberdoerffer P, Sandberg R and Oberdoerffer S: CTCF-promoted RNA polymerase II pausing links DNA methylation to splicing. Nature 479: 74-79, 2011.

57. Paredes SH, Melgar MF and Sethupathy P: Promoter-proximal CCCTC-factor binding is associated with an increase in the transcriptional pausing index. Bioinformatics 29: 1485-1487, 2013

58. Adelman K and Lis JT: Promoter-proximal pausing of RNA polymerase II: Emerging roles in metazoans. Nat Rev Genet 13: 720-731, 2012.

59. Ong CT and Corces VG: CTCF: An architectural protein bridging genome topology and function. Nat Rev Genet 15: 234-246, 2014.

60. Stadhouders R, Thong juea S, Andrieu-Soler C, Palstra RJ, Bryne JC, van den Heuvel A, Stevens M, de Boer E, Kockx C, van der Sloot A, et al: Dynamic long-range chromatin interactions control Myb proto-oncogene transcription during erythroid development. EMBO J 31: 986-999, 2012.

61. Sebestyén E, Zawisza M and Eyras E: Detection of recurrent alternative splicing switches in tumor samples reveals novel signatures of cancer. Nucleic Acids Res 43: 1345-1356, 2015.

62. Ulaner GA, Hu JF, Vu TH, Giudice LC and Hoffman AR Tissue-specific alternate splicing of human telomerase reverse transcriptase (hTERT) influences telomere lengths during human development. Int J Cancer 91: 644-649, 2001.

63. Lincz LF, Mudge LM, Scorgie FE, Sakoff JA, Hamilton CS and Seldon M: Quantification of hTERT splice variants in melanoma by SYBR green real-time polymerase chain reaction indicates a negative regulatory role for the $\beta$ deletion variant. Neoplasia 10 $1131-1137,2008$

64. Mavrogiannou E, Strati A, Stathopoulou A, Tsaroucha EG, Kaklamanis L and Lianidou ES: Real-time RT-PCR quantification of human telomerase reverse transcriptase splice variants in tumor cell lines and non-small cell lung cancer. Clin Chem 53 53-61, 2007.

65. Listerman I, Sun J, Gazzaniga FS, Lukas JL and Blackburn EH: The major reverse transcriptase-incompetent splice variant of the human telomerase protein inhibits telomerase activity but protects from apoptosis. Cancer Res 73: 2817-2828, 2013.

66. Kolquist KA, Ellisen LW, Counter CM, Meyerson M, Tan LK, Weinberg RA, Haber DA and Gerald WL: Expression of TERT in early premalignant lesions and a subset of cells in normal tissues. Nat Genet 19: 182-186, 1998.

67. Meyerson M, Counter CM, Eaton EN, Ellisen LW, Steiner P, Caddle SD, Ziaugra L, Beijersbergen RL, Davidoff MJ, Liu Q, et al: hEST2, the putative human telomerase catalytic subunit gene, is up-regulated in tumor cells and during immortalization. Cell 90: 785-795, 1997.

68. Yi X, Shay JW and Wright WE: Quantitation of telomerase components and hTERT mRNA splicing patterns in immortal human cells. Nucleic Acids Res 29: 4818-4825, 2001.
69. Kotoula V, Hytiroglou P, Pyrpasopoulou A, Saxena R, Thung SN and Papadimitriou CS: Expression of human telomerase reverse transcriptase in regenerative and precancerous lesions of cirrhotic livers. Liver 22: 57-69, 2002.

70. Ohyashiki JH, Hisatomi H, Nagao K, Honda S, Takaku T, Zhang Y, Sashida G and Ohyashiki K: Quantitative relationship between functionally active telomerase and major telomerase components (hTERT and hTR) in acute leukaemia cells. Br J Cancer 92: 1942-1947, 2005.

71. Barclay JY, Morris AG and Nwokolo CU: HTERT mRNA partially regulates telomerase activity in gastric adenocarcinoma and adjacent normal gastric mucosa. Dig Dis Sci 50: 1299-1303, 2005.

72. Rha SY, Jeung HC, Park KH, Kim JJ and Chung HC: Changes of telomerase activity by alternative splicing of full-length and $\beta$ variants of hTERT in breast cancer patients. Oncol Res 18: 213-220, 2009.

73. Liu Y, Wu BQ,Zhong HH, Tian XX and Fang WG: Quantification of alternative splicing variants of human telomerase reverse transcriptase and correlations with telomerase activity in lung cancer. PLoS One 7: e38868, 2012.

74. Wang Y, Meeker AK, Kowalski J, Tsai HL, Somervell H, Heaphy C, Sangenario LE, Prasad N, Westra WH, Zeiger MA, et al: Telomere length is related to alternative splice patterns of telomerase in thyroid tumors. Am J Pathol 179: 1415-1424, 2011

75. Wong MS, Wright WE and Shay JW: Alternative splicing regulation of telomerase: A new paradigm? Trends Genet 30: 430-438, 2014.

76. Kilian A, Bowtell DD, Abud HE, Hime GR, Venter DJ, Keese PK, Duncan EL, Reddel RR and Jefferson RA: Isolation of a candidate human telomerase catalytic subunit gene, which reveals complex splicing patterns in different cell types. Hum Mol Genet 6: 2011-2019, 1997.

77. Saebøe-Larssen S, Fossberg E and Gaudernack G: Characterization of novel alternative splicing sites in human telomerase reverse transcriptase (hTERT): Analysis of expression and mutual correlation in mRNA isoforms from normal and tumour tissues. BMC Mol Biol 7: 26, 2006.

78. Withers JB, Ashvetiya T and Beemon KL: Exclusion of exon 2 is a common mRNA splice variant of primate telomerase reverse transcriptases. PLoS One 7: e48016, 2012.

79. Khosravi-Maharlooei M, Jaberipour M, Hosseini Tashnizi A, Attar A, Amirmoezi F and Habibagahi M: Expression pattern of alternative splicing variants of human telomerase reverse Transcriptase (hTERT) in cancer cell lines was not associated with the origin of the cells. Int J Mol Cell Med 4: 109-119, 2015.

80. Jafri MA, Ansari SA, Alqahtani MH and Shay JW: Roles of telomeres and telomerase in cancer, and advances in telomerasetargeted therapies. Genome Med 8: 69, 2016. 